\title{
Improved Vision-Based Detection of Strawberry Diseases Using a Deep Neural Network
}

\author{
Byoungjun Kim¹, You-Kyoung Han², Jong-Han Park² and Joonwhoan Lee ${ }^{1 *}$ \\ ${ }^{1}$ Division of Computer Science and Engineering, Jeonbuk National University, Jeonju, South Korea, ${ }^{2}$ Horticultural and Herbal \\ Crop Environment Division, National Institute of Horticultural and Herbal Science (RDA), Jeonju, South Korea
}

OPEN ACCESS

Edited by:

Roger Deal,

Emory University, United States

Reviewed by:

Jin Chen,

University of Kentucky, United States

Francesco Cellini,

Agenzia Lucana di Sviluppo e di Innovazione in Agricoltura (ALSIA),

Italy

*Correspondence:

Joonwhoan Lee chlee@jbnu.ac.kr

Specialty section:

This article was submitted to Technical Advances in Plant Science, a section of the journal

Frontiers in Plant Science

Received: 05 May 2020

Accepted: 25 November 2020

Published: 11 January 2021

Citation:

Kim B, Han Y-K, Park J-H and Lee J (2021) Improved Vision-Based Detection of Strawberry Diseases

Using a Deep Neural Network.

Front. Plant Sci. 11:559172.

doi: $10.3389 /$ fp/s.2020.559172
Detecting plant diseases in the earliest stages, when remedial intervention is most effective, is critical if damage crop quality and farm productivity is to be contained. In this paper, we propose an improved vision-based method of detecting strawberry diseases using a deep neural network (DNN) capable of being incorporated into an automated robot system. In the proposed approach, a backbone feature extractor named PlantNet, pre-trained on the PlantCLEF plant dataset from the LifeCLEF 2017 challenge, is installed in a two-stage cascade disease detection model. PlantNet captures plant domain knowledge so well that it outperforms a pre-trained backbone using an ImageNet-type public dataset by at least 3.2\% in mean Average Precision (mAP). The cascade detector also improves accuracy by up to $5.25 \% \mathrm{mAP}$. The results indicate that PlantNet is one way to overcome the lack-of-annotated-data problem by applying plant domain knowledge, and that the human-like cascade detection strategy effectively improves the accuracy of automated disease detection methods when applied to strawberry plants.

Keywords: strawberry diseases, cascade detector, deep neural network, detection, plant domain knowledge

\section{INTRODUCTION}

Effectively protecting plants from diseases is a critical means of improving productivity and enhancing crop quality (Fuentes et al., 2018). The traditional methods for the identification and diagnosis of plant diseases - visual analysis by professional farmer or inspection of a sample in a laboratory - generally require extensive professional knowledge and high costs. Moreover, neither method is particularly effective, with carrying a high probability of failure in successfully diagnosing specific diseases, leading to erroneous conclusions and treatments (Ferentinos, 2018).

Detecting plant diseases in their earliest stages can reduce the need to rely on potentially harmful remedial chemicals and lower labor costs. As many greenhouses are quite large, it is not always easy for even the most experienced farmers to identify plant diseases before they have spread. For this reason, an automated disease detection process will prove to be a valuable supplement to the labor and skill of farmers.

Researchers have already developed automatic identification and diagnosis methods capable of reaching fast, convenient, and accurate conclusions based on image analysis and machine learning techniques. Currently, there are two types of vision-based disease detection methods. 
First, are those methods that require humans to be kept in the detection loop. For example, the PlantVillage dataset (Hughes and Salathé, 2015) consists of images of detached leaves. When a detection system trained with a dataset like this is installed on handheld mobile devices equipped with a camera, a human is expected to take the initiative in identifying suspicious leaves and running the app. In the second type of system, crop monitoring robots capable of surveilling the whole of a crop at once are installed throughout a greenhouse. The images collected by these robots can be analyzed to identify a potential disease and automatically alert the appropriate supervisor. While such a surveillance system presents many logistical challenges in migrating from the drawing-board to the greenhouse, detection model is highly desirable for its superior early detection abilities and potential for cost savings.

The typical farmer approaches disease detection in two stages. First, he or she tries to identify suspicious areas on a plant that, based on his (or her) domain knowledge, may indicate disease. They then must determine whether it is a real disease or not. This paper proposes an improved deep learning-based strawberry disease detection method that relies on automatic visual object detection to imitate the two-steps of the human expert-detection process. First, our proposed system uses domain knowledge specific about plants for transfer learning. Second, it relies on a cascade detection scheme, by which it first glances over the objects in order to identify large suspicious regions, and then scrutinizes the suspicious area more closely to precisely identify the disease.

In general, plants vary in the shapes and colors of their flowers, leaves, stems, fruits, and roots. In light of this great diversity, it is inappropriate to analyze and extract plant features using a pre-trained model based on the ImageNet dataset, as this dataset does not reflect adequate domain knowledge. Typically, source and target domains are well connected with each other, which maximizes the effect of transfer learning. This explains why it is so inefficient to use pre-trained models with coarse-grained public datasets like ImageNet (Krizhevsky et al., 2012) for plant disease detection or classification.

Our proposed method uses networks named PlantNets, pre-trained with the PlantCLEF dataset from the LifeCLEF 2017 challenge (Joly et al., 2017), to construct a deep object detection model by transfer learning. Unlike prior disease detection or classification approaches (Kawasaki et al., 2015; Mohanty et al., 2016; Sladojevic et al., 2016; Brahimi et al., 2017; Cruz et al., 2017; Fuentes et al., 2017, 2018, 2019; Ramcharan et al., 2017, 2019; Barbedo, 2018; Ferentinos, 2018; Liu et al., 2018; Nie et al., 2019; Too et al., 2019), we assume a PlantNet backbone network pre-trained on the PlantCLEF dataset has the low-level domain knowledge of a farmer experienced at plant and disease identification.

The proposed method then uses a two-stage cascade detection model. In the first stage, the system surveilles a large visual field, seeking visual symptoms of diseases or other objects that may possibly cause a false positive. In the second stage, the system closely checks only the disease-suspected area to improve diagnosis and precision. In this way, the system reduces false positives caused by adverse environmental factors, like glare or other objects.

In our experimental a single-stage detector with a PlantNet provided an $86.4 \% \mathrm{mAP}$ in the complex task of strawberry disease detection, which represents a $3.27 \%$ improvement over a model pre-trained on the ImageNet dataset. In addition, the cascade model produced approximately $91.65 \% \mathrm{mAP}$, which represents a $5.25 \%$ gain over a non-cascade model.

The remainder of this paper is organized as follows. In the next section, we provide a detailed review of previous works related to our method. Further details about the cascade strategy for disease detection with a PlantNet are provided in Section 3. In Section 4 we outline the proposed approach to strawberry disease detection. Our experimental results are presented in Section 5, along with a discussion of the performance of our method. Finally, in Section 6 we summarize our work, and suggest what new lines of inquiry have been opened by our research.

\section{RELATED WORKS}

A conventional computer vision approach consists of feature exploration by image analysis and identifier construction by machine learning. Many visual feature classifiers have been developed for application in many problem domains (Zhao et al., 2019). In general, for traditional computer vision, there have been many expert-designed features descriptors developed, including the Scale Invariant Feature Transform (SIFT) (Lowe, 2004), the Haar (Viola and Jones, 2001), and the Histogram of Oriented Gradients (HOG) (Dalal and Triggs, 2005). Theses have been exploited with machine learning algorithms, such as the Support Vector Machine (SVM), to construct detectors or classifiers.

The deep learning approach differs from the conventional computer vision-based disease recognition in several way. First, the features are not selected by an expert, but are automatically derived by a network relying on a large amount of training data. In designing this approach, therefore, the goal is not to identify the proper features, but rather to finding an appropriate network and preparing suitable training data. Another difference is that the detector or the classifier can be obtained by simultaneously training it with features in the same deep neural network. Therefore, the appropriate network structure becomes more important with an efficient training algorithm.

There are two different vision-based approaches to plant disease recognition by machines: classification and detection. The classification approach relies on a human to visually identify symptoms of a plant disease, and then capture an image and ask a computer to provide a diagnosis. This approach starts with an image of part or whole of a plant canopy, diseased or healthy, and then follows this with an attempt determine whether the image includes symptoms of a specific disease.

The detection approach, in contrast, first tries to identify the location of symptoms, usually within bounding boxes, and classify them as specific diseases. This process is localized to, suspicious areas to reduce the necessity of human intervention. In this system, an image in which symptoms are detected 
results in an output of bounding boxes with the corresponding names of diseases. Input images for the detection approach are generally less restricted than those of the classification approach, as they can be captured from any direction or distance to reveal the symptoms. The detection approach can also be applied to a sequence of video frames, while the classification approach typically relies on only a single image. Being the more flexible and comprehensive of the two options, it comes as no surprise that a detection system was preferred in designing our automated system.

\section{Classification Approaches for Disease Identification}

There have been a number of prior Deep Neural Network (DNN)-based classification approaches to plant diseases and disorder identification. Such DNNs usually consists of a multilayer Convolutional Neural Network (CNN)-based feature representation block which is followed by a classification block. The PlantVillage dataset has been widely used to train classifiers because of its excellent images of plant disease.

Table 1 summarizes recent research in which Deep Learning (DL)-based classification approaches have been applied. Note that a great deal of the research used the ImageNet pre-trained backbone models as a feature extractor, and the PlantVillage dataset to fine-tune the specific feature extractor and classifier to plants and diseases. In addition, (Park et al., 2018) proposed a DL-based classification approach for the five types of apple leaf conditions in hyperspectral images for diagnosis of Marssonina blotch, in which they selected several bands by minimum redundancy maximum relevance (mRMR). Also, (You and Lee, 2020) developed a lightweight MobileNet-based DNN for classifying citrus diseases and pests.

\section{Detection Approaches for Disease Identification}

In general, DL-based visual object detections are divided into single-step and multi-step approaches. OverFeat (Sermanet et al., 2013) was one of the first single-stage object detections based on deep learning networks. There are two primary means of applying the single-step approach; Single Shot Multi-Box Detector (SSD) (Liu et al., 2016) and You Only Look Once (YOLO) (Redmon et al., 2016). The single-step approach reduces the computational complexity of the network to improve detection speed, which is its primary advantage over the multistep approach. Plant disease detections by DL-based computers that use the single-step approach have been developed for various spices, such as cassava (Ramcharan et al., 2017, 2019) and tomatoes (Fuentes et al., 2017, 2018, 2019).

The multi-step approach is based on a two-step process. The first step generates a set of candidate region proposals (Uijlings et al., 2013), and the second step classifies the proposals into either foreground (enclosed by bounding boxes) or background. This approach originated from the Region-based Convolutional Neural Network (R-CNN) (Girshick et al., 2014) and successive improvements in detection speed and accuracy led to the Fast R-CNN (Girshick, 2015), the Faster R-CNN (Ren et al., 2015), the Region-based Fully Convolutional Network (R-FCN) (Dai et al., 2016), and the Feature Pyramid Network (FPN) (Lin et al., 2017). Several plant disease detection models have used the multi-stage approach to identify, for example, verticillium wilt in strawberries (Nie et al., 2019), and diverse diseases in tomatoes (Fuentes et al., 2017, 2018, 2019). Table 2 summarizes plant disease identification based on visual object detection.

As gathering and labeling data for disease identification is expensive, all of the prior research used pre-trained networks and applied transfer learning. Gathering even annotated data for object detection is much harder than it is for classification, as bounding boxes must be provided for ground truth. In general, all DNN-based object detection methods adopted the backbone networks pre-trained on the ImageNet dataset of the ImageNet Large Scale Visual Recognition Challenge (ILSVRC) or the Microsoft Common Objects in Context (MS COCO) dataset (Lin et al., 2014), which is appropriate for coarse-grained identification tasks. Because there is no public dataset like PlantVillage for classification, every detection model developed has used its own dataset collected from the field to finetune the network.

\section{THE PROPOSED APPROACH}

In light of the many advantages of the object detection model for automating early monitoring for plant diseases, our DLbased approach adopts this approach. In our two-stage cascade structure, the initial diagnosis stage identifies suspected diseased area and the second stage is used for fine detection. Each stage is constructed with one of the PlantNets as a backbone for the feature extractor, which is pre-trained using the PlantCLEF dataset from the LifeCLEF 2017 challenge.

The scope of this work does not include abiotic stresses. We assume that strawberry growing in relatively small sized greenhouses in South Korea, where the abiotic stresses are relatively rare and the image capturing is less hard to be incorporated with a robot system.

\section{Plant Domain Knowledge Network (PlantNet)}

Detecting disease on the basis of plant images is not a coarsegrained task like ILSVRC, which includes objects such as elephants, cars, apples, etc., and which all look different from each other. Many plant diseases and disorders look very similar, so even an expert might struggle to correctly diagnose and remediate the symptoms. Accordingly, the backbone networks pre-trained with an ImageNet dataset may not be sufficient to be applied to the tack of detecting disease from plant images.

Successful transfer learning requires that, the visual features in the target domain should be properly transformed from those of the source domain by fine-tuning, even with a small training dataset. Labeled data for plant disease detection is expensive to acquire, as the name and location of a disease must be annotated with the corresponding bounding box by a domain expert. Accordingly, it was inevitable that we would have 
TABLE 1 | L-based classification approaches for identification of plant diseases.

\begin{tabular}{|c|c|c|c|c|c|}
\hline Authors & Network Models & Dataset for pre-training & Plants & Dataset for fine-tuning & Disease classes \\
\hline Sladojevic et al., 2016 & CaffeNet & ImageNet & 15 spices & Collected from Internet & 13 \\
\hline Cruz et al., 2017 & Modified LeNet & PlantVillage & olives & PlantVillage & 3 \\
\hline Mohanty et al., 2016 & AlexNet, GoogLeNet & ImageNet & 14 spices & PlantVillage & 38 \\
\hline Barbedo, 2018 & GoogLeNet & ImageNet & 12 spices & & 12 \\
\hline Too et al., 2019 & $\begin{array}{l}\text { VGG16, InceptionV4, } \\
\text { ResNet50, -101, -152, } \\
\text { DenseNet121 }\end{array}$ & ImageNet & 14 spices & PlantVillage & 38 \\
\hline Brahimi et al., 2017 & AlexNet, GoogLeNet & ImageNet & 9 diseases & PlantVillage & 9 \\
\hline Ramcharan et al., 2017 & InceptionV3 & ImageNet & cassava & Collected from fields & 5 \\
\hline Kawasaki et al., 2015 & Modified LeNet & & cucumber & Collected from laboratory & 3 \\
\hline Ferentinos, 2018 & $\begin{array}{l}\text { AlexNet, GoogLeNet, OverFeat, } \\
\text { VGG16, AlexNetOWTBn }\end{array}$ & & 25 species & PlantVillage & 58 \\
\hline Liu et al., 2018 & AlexNet & ImageNet & apple & Collected from fields & 4 \\
\hline
\end{tabular}

TABLE 2 | Summary of visual object detection models for plant disease monitoring.

\begin{tabular}{|c|c|c|c|c|c|}
\hline Authors & Models & Dataset for pre-training & Plants & Dataset for fine-tuning & No. of classes \\
\hline Nie et al., 2019 & Faster R-CNN + Attention & ImageNet & strawberry & Collected from fields & 4 \\
\hline Ramcharan et al., 2019 & SSD & MSCOCO & cassava & Collected from fields & 3 \\
\hline Fuentes et al., 2017 & Faster R-CNN,R-FCN, SSD & ImageNet & tomato & Collected from fields & 9 \\
\hline Fuentes et al., 2018 & Faster R-CNN + FilterBank & ImageNet & tomato & Collected from fields & 10 \\
\hline Fuentes et al., 2019 & FPN + LSTM & ImageNet & tomato & Collected from fields & 10 \\
\hline
\end{tabular}

to overcome a lack-of-training-data problem in our efforts to achieve accurate detection.

Because no large image dataset for plant diseases exists, we relied on the PlantCLEF dataset from the LifeCLEF 2017 challenge, which was designed to study biodiversity and agrobiodiversity (Joly et al., 2017). We believe PlantCLEF is superior to ImageNet for extracting the proper features of plant diseases, as these images are particularly well-suited successfully fine-tune for plant disease detection with a small amount of domain-specific, annotated training data.

The PlantCREF dataset includes images of weeds, trees, ferns, etc., and their various parts, including the flowers, fruits, and leaves. The dataset includes 256,278 credible and noiseless plant images, and approximately $1.45 \mathrm{M}$ images with noise gathered off the internet. Figure 1 shows several sample images taken from the noiseless dataset. We filtered out thousands of noisy images, and retrained approximately $1.25 \mathrm{M}$ images.

We tested three recent backbone networks, including ResNet50, ResNet152, and the InceptionResNet v2 models, to do the classification task of the LifeCLEF 2017 challenge. We ultimately choose ResNet152, which gave the best performance with respect to Top-5 classification accuracy.

\section{Cascade Strawberry Diseases Detection Model}

Our strawberry disease detection model adopts a two-step cascade object detection technique. Figure 2 shows the proposed detection model. The first stage detects suspicious areas of diseases with a low threshold-of-confidence score for the locations of the bounding boxes. In general, the role of the first stage in cascade detection is to increase the recall rate by using a low detection threshold. Improving precision and reducing false detections is the goal of the second stage. In the first stage, we defined three categories; "normal," "abnormal (fruit, leaf)," and "background object" (to include items such as vinyl or other items that are not part of the plant). Figure 3 shows the detection and merged results of the first stage. Adjacent suspicious areas of disease (a short distance apart) were then merged as rectangles to prepare a single large area. In this merging process the detected background objects were excluded so that they would not be examined in the second stage. This process removed areas where diseases would not manifest in order to reduce false detections and reduce speed in reaching the second stage.

The merged area of the rectangle was cropped and resized to feed into the second stage module, where suspicious areas were again scrutinized to more precisely detect seven categories of six diseases, including "angular leafspot," "anthracnose fruit rot," "gray mold," "leaf blight," "leaf spot," and "powdery mildew fruit/leaf." The final results of the analysis of disease class and locations are displayed in the input image. The second stage review provides high accuracy and low false detection rates by carefully observing only the confined areas. Note that the structure of the detection modules are identical even though the object classes to be detected are different from each other.

\section{Strawberry Disease Detection Modules}

Because the ResNet152 backbone network was the best performer at the LifeCREF 2017 classification task, it was chosen as the initial backbone feature extractor for both the first and second detection modules. Once the features are extracted, they are captured in an FPN structure. Based 

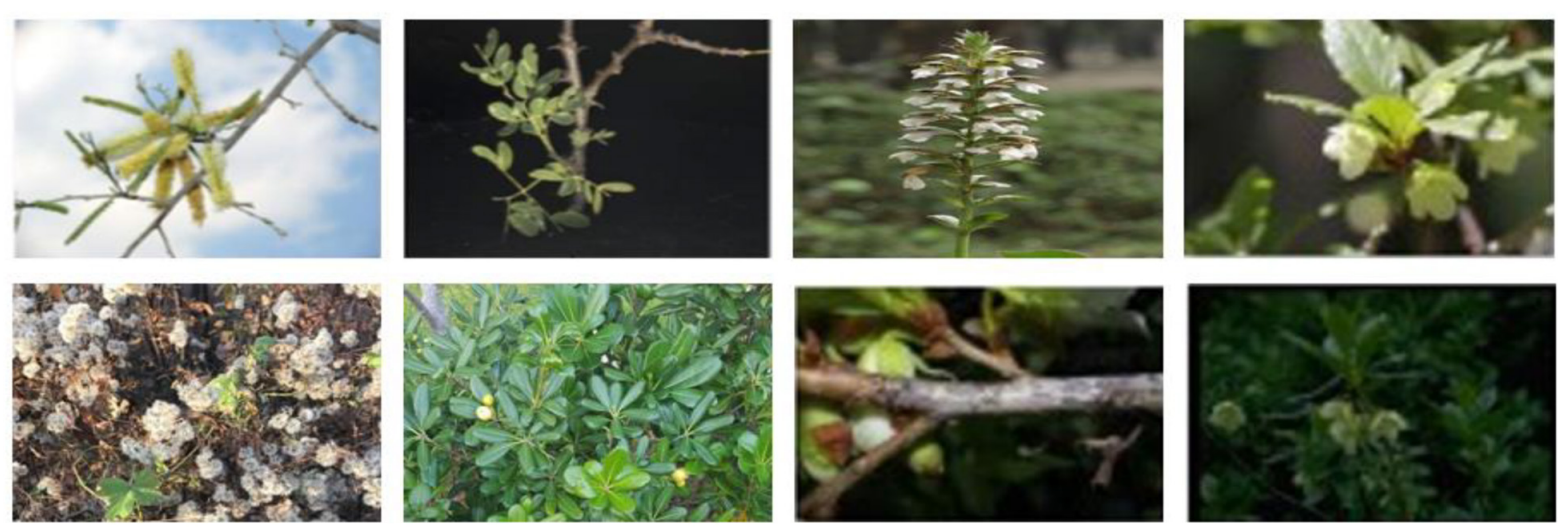

FIGURE 1 | Examples of noiseless samples from the PlantCLEF dataset in LifeCLEF 2017 challenge (Joly et al., 2017).

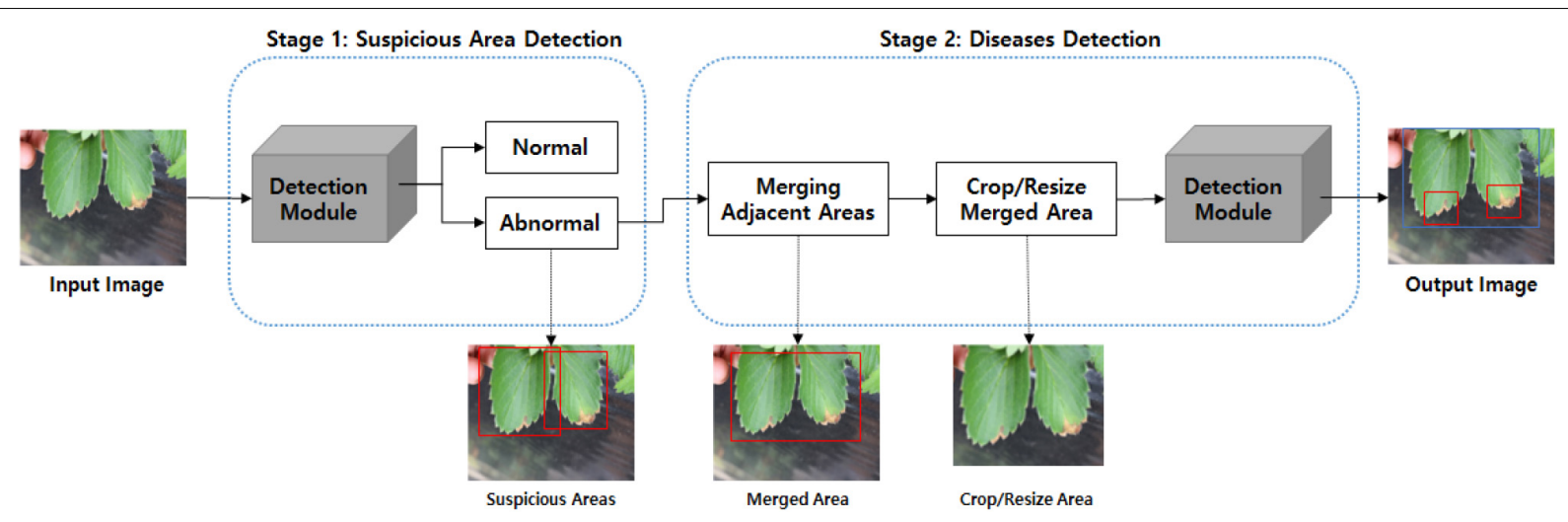

FIGURE 2 | Proposed strawberry disease detection model.
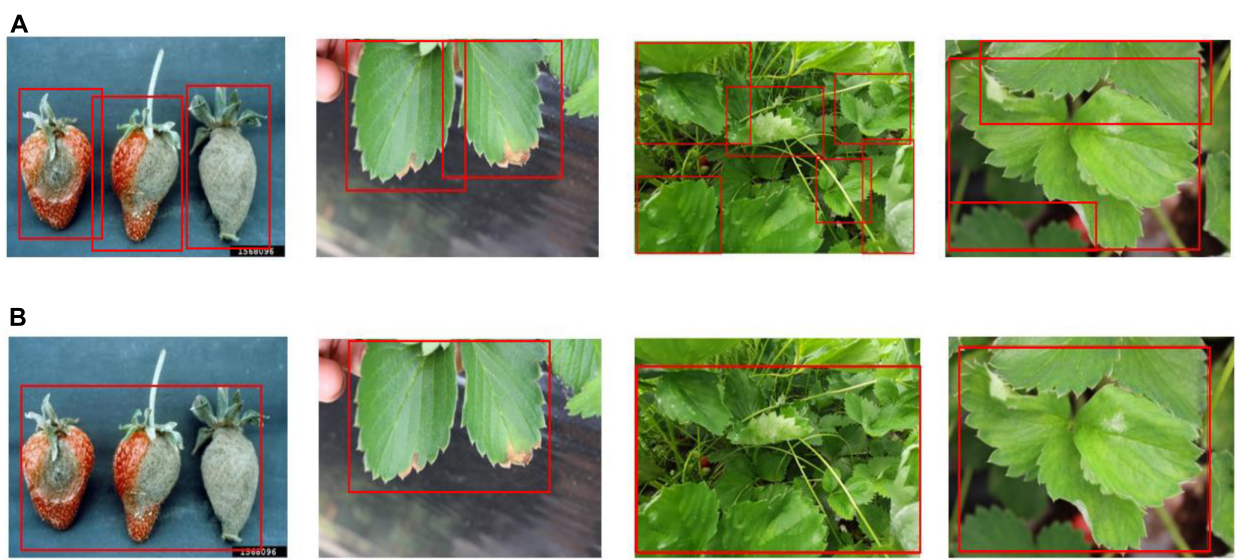

FIGURE 3 | Detection and merged results of the first stage: (A) suspicious areas of disease in the first stage, and (B) merged areas for input to the second stage. [Forestry Images (2020); photographer: Sikora].

on this multi-scale feature representation, the regions to be classified are proposed and precisely located. Figure 4 shows the ResNet152 backbone and the FPN-structured disease detection module.

\section{EXPERIMENTS}

In this section, we present the experimental results for the PlantNets, and our collected strawberry dataset. 


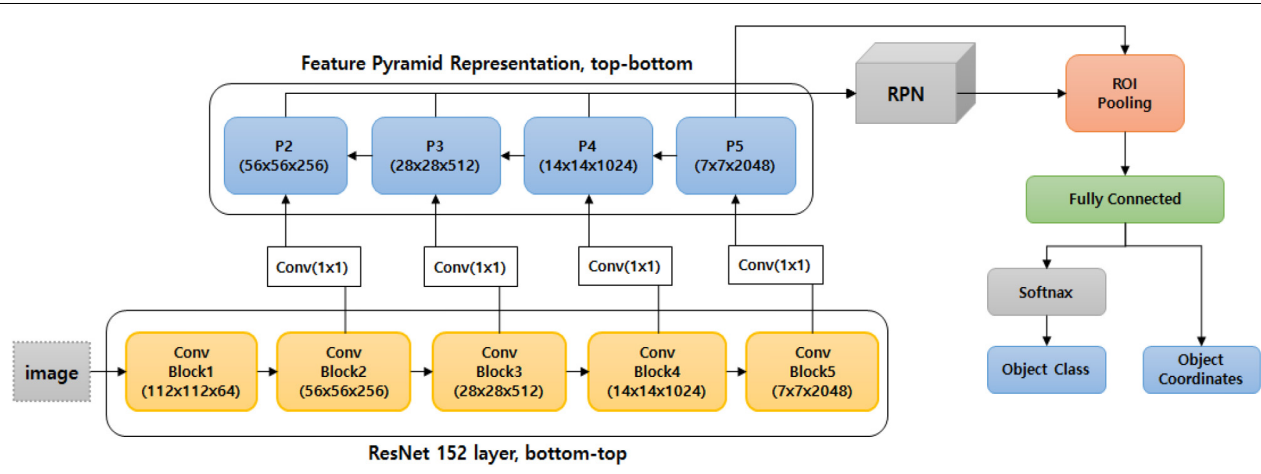

FIGURE 4 | ResNet152 backbone and the FPN-structured disease detection module.

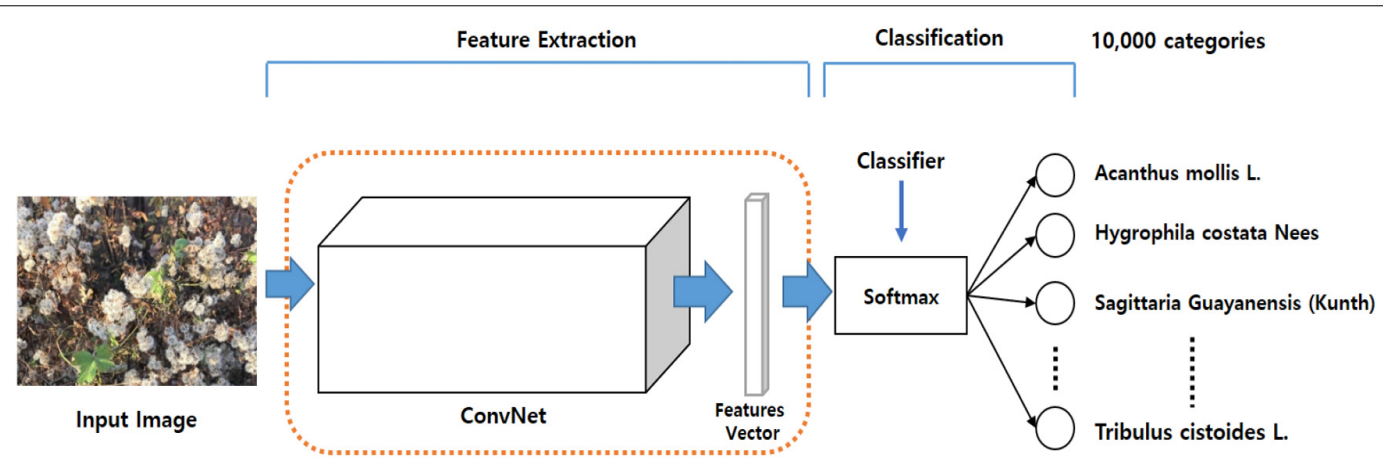

FIGURE 5 | LifeCLEF 2017 task for a round dotted red-rectangle PlantNet.

TABLE 3 | Summary of results from the LifeCLEF 2017 Task.

\begin{tabular}{|c|c|c|c|c|}
\hline \multirow[t]{2}{*}{ Backbone } & \multirow[t]{2}{*}{ Accuracy } & \multirow[t]{2}{*}{ Features obtained from ImageNet dataset } & \multicolumn{2}{|c|}{ Fine-tuned by PlantCLEF 2017 dataset } \\
\hline & & & 250K noise-free data & $1.50 \mathrm{M}+$ noisy data \\
\hline \multirow[t]{2}{*}{ ResNet50 } & Top-1 & $20.80 \%$ & $51.70 \%$ & $69.60 \%$ \\
\hline & Top-5 & $26.30 \%$ & $79.50 \%$ & $85 \%$ \\
\hline \multirow[t]{2}{*}{ ResNet152 } & Top-1 & $27.10 \%$ & $64.80 \%$ & $76.30 \%$ \\
\hline & Top-5 & $32.80 \%$ & $84.00 \%$ & $91.00 \%$ \\
\hline \multirow[t]{2}{*}{ InceptionResNetV2 } & Top-1 & $24.50 \%$ & $67.50 \%$ & $75.50 \%$ \\
\hline & Top-5 & $38.00 \%$ & $83.50 \%$ & $90.00 \%$ \\
\hline
\end{tabular}

\section{LifeCLEF 2017 Task and PlantNets}

To demonstrate the necessity for transfer learning, and to identify the backbone network best suited to capturing visual features from plant images, we followed the LifeCLEF 2017 task, which constructs an image classifier for 10,000 categories of different plants, as shown in Figure 5.

In our experiment, we first comprise the backbone networks with the parameters trained on the ImageNet dataset and adjusted the parameters associated with the Softmax classifier for the 10,000 categories. Note that this experiment relied on only the learned feature extractor that was trained on the ImageNet dataset, and only attempted to train the classifier to perform the task. This setting resulted in poor performance, as expected, by the ResNet50, ResNet152, and InceptionResNetV2, as listed in Table 3.

The backbone was then pre-trained with the ImageNet dataset and the Softmax classifier was fine-tuned with the LifeCLEF 2017 dataset. Performance was greatly improved by fine-tuning both the backbone feature extractor and the classifier network, suggesting that fine-tuning is necessary prerequisite to improving performance with domain-specific data. Performance improved as the amount of data increased, even when noisy data was included. Among the Top- 5 categories, ResNet152 was the best among the three, providing $91.0 \%$ classification accuracy, as shown in Table 3. Based on these results, we chose ResNet152 as the backbone of the strawberry disease detector. The dotted red rounded rectangle in Figure 5 represents the PlantNet. 
TABLE 4 | Number of images for strawberry disease detection.

\begin{tabular}{|c|c|c|c|c|}
\hline $\begin{array}{l}\text { Categories of positive and } \\
\text { negative examples }\end{array}$ & Original number of images & $\begin{array}{l}\text { Images for training and } \\
\text { validation }\end{array}$ & $\begin{array}{c}\text { Augmented number of } \\
\text { images for training and } \\
\text { validation }\end{array}$ & $\begin{array}{c}\text { Numbers of test } \\
\text { images for evaluation }\end{array}$ \\
\hline Angular leafspot & 319 & 223 & 2676 & 96 \\
\hline Anthracnose fruit rot & 231 & 161 & 1932 & 70 \\
\hline Gray mold & 235 & 164 & 1968 & 71 \\
\hline Leaf blight & 294 & 209 & 2508 & 85 \\
\hline Leaf spot & 593 & 415 & 4980 & 178 \\
\hline Powdery mildew fruit & 137 & 97 & 1164 & 40 \\
\hline Powdery mildew leaf & 718 & 502 & 6024 & 216 \\
\hline Normal & 2000 & 1400 & 16800 & 600 \\
\hline Background & 198 & 138 & 1656 & 60 \\
\hline Total & 4725 & 3309 & 39708 & 1416 \\
\hline
\end{tabular}
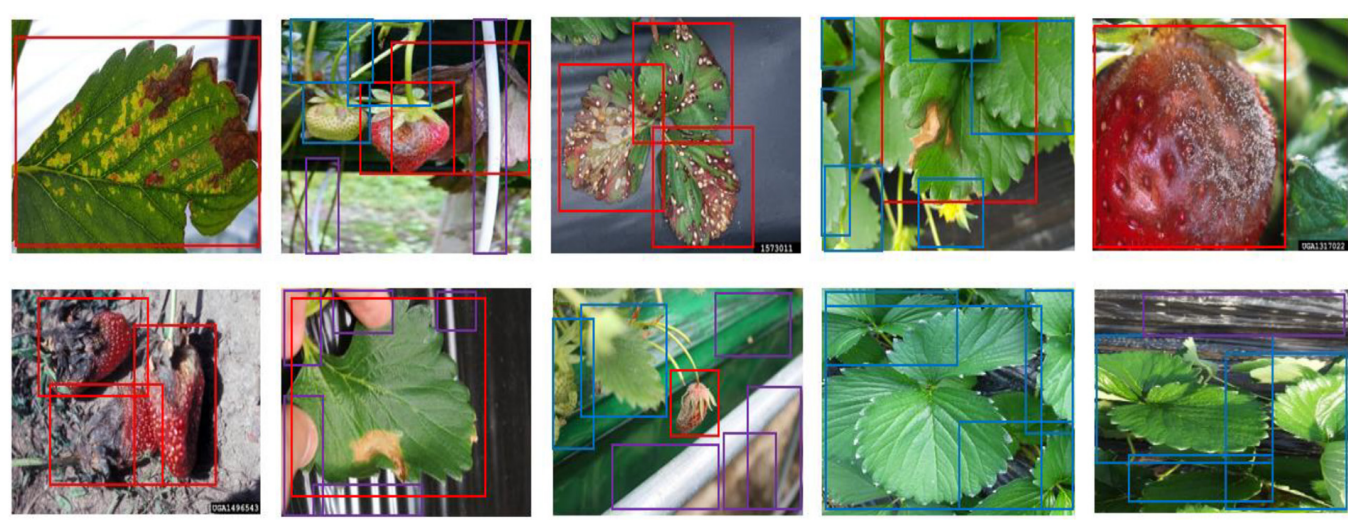

FIGURE 6 | The annotated sample images from the collected dataset. Ground truth is annotated by bounding boxes with the following class categories: diseases (red box), normal plants (blue box), and background object (purple box).

\section{Strawberry Dataset}

We collected 4,560 images from different greenhouses using camera-equipped mobile phones, and combine them with 175 Forestry Images $^{1}$ for a total 4,735 images as in Table 4 .

${ }^{1}$ Forestry Images (2020), https://www.forestryimages.org/index.cfm (accessed November 7, 2020). Photographers: Brenda Kennedy, University of Kentucky, Bugwood.org; Bruce Watt, University of Maine, Bugwood.org; Clemson University

TABLE 5 | Comparison of results from ImageNet- and PlantNet-pre-trained backbones in a single-stage detector.

\begin{tabular}{lcc}
\hline Class & $\begin{array}{c}\text { Average precision of the } \\
\text { ImageNet pre-trained } \\
\text { backbone }\end{array}$ & $\begin{array}{c}\text { Average precision of } \\
\text { PlantNet }\end{array}$ \\
\hline Angular leafspot & $74.72 \%$ & $81.69 \%$ \\
Anthracnose fruit rot & $87.38 \%$ & $90.23 \%$ \\
Gray mold & $89.68 \%$ & $90.00 \%$ \\
Leaf blight & $87.64 \%$ & $89.81 \%$ \\
Leaf spot & $71.66 \%$ & $80.48 \%$ \\
Powdery mildew fruit & $83.94 \%$ & $84.01 \%$ \\
Powdery mildew leaf & $86.91 \%$ & $88.59 \%$ \\
mAP & $83.13 \%$ & $86.40 \%$
\end{tabular}

We assumed that the images were collected from diverse environmental conditions for strawberries, as they were taken

- USDA Cooperative Extension Slide Series, Bugwood.org; Don Ferrin, Louisiana State University Agricultural Center, Bugwood.org; Edward Sikora, Auburn University, Bugwood.org; Florida Division of Plant Industry, Florida Department of Agriculture and Consumer Services, Bugwood.org; Gerald Holmes, Strawberry Center, Bugwood.org; Garrett Ridge, NCSU, Bugwood.org; Jonas Janner Hamann, Universidade Federal de Santa Maria (UFSM), Bugwood.org; Ko Ko Maung, Bugwood.org; Mary Ann Hansen, Virginia Polytechnic Institute and State University, Bugwood.org; Natalie Hummel, Louisiana State University AgCenter, Bugwood.org; Paul Bachi, University of Kentucky Research and Education Center, Bugwood.org; Rebecca A. Melanson, Mississippi State University Extension, Bugwood.org; Scott Bauer, USDA Agricultural Research Service, Bugwood.org; U. Mazzucchi, Università di Bologna, Bugwood.org.

TABLE 6 | Comparison of results from the loU detection threshold in the first-stage detector.

\begin{tabular}{lccc}
\hline loU threshold & True Positive & False Negative & Recall \\
\hline 0.1 & 39708 & 0 & 1 \\
0.2 & 39578 & 130 & 0.9941976 \\
0.3 & 39492 & 216 & 0.989226633 \\
0.4 & 39333 & 375 & 0.983649467 \\
0.5 & 38160 & 1548 & 0.961471133
\end{tabular}


different farms. The dataset included images of the beginning, middle, and final stages of various diseases, as well as unblemished strawberries. Such diversity in the dataset is important to successfully train a generalized disease detector. Figure 6 shows sample images from the dataset.

\section{Training the Strawberry Disease Detector}

The dataset was divided into training, validation, and test datasets. About $60 \%$ of the images in the dataset were taken for training, and $10 \%$ were used for validation. To reduce the chance of overfitting, we augmented the training and validation dataset with geometric transformations, including resized, cropped, rotated, horizontal/vertical flipped, and PCA color-changed images. The test dataset used evaluation was not augmented. Table 4 describes the training, validation, and testing datasets in greater detail. We separated the background, which included objects like pillars that supported the greenhouse and black vinyl to protect against weeds, from the foreground images of the strawberry plant. The normal category includes images of healthy strawberries, which corresponds to the negative examples as well as background in the first stage of cascade detection. Seven different categories of strawberry diseases were considered (powdery mildew has two different categories, depending on where it manifests on the plant). As shown in Figure 6, ground truth is annotated by bounding boxes with the following class categories: diseases (red box), normal plants (blue box), and background object (purple box). From the images listed in Table 4, we annotated 21,252 bounding boxes for abnormal classes (positive examples of diseases and background objects) and 16,800 for normal classes (negative examples, i.e., normal strawberries).

Training was performed separately for each detection module. Because this includes merging the bounding boxes and the crop/resizing process that prepares the input for the second stage of the detection module, end-to-end learning was not possible. The proposed model was trained and evaluated on an Intel Xeon CPU E5-2650, 64GB RAM, and a single NVidia Geforce TiTanXP GPU. The model parameters were selected as follows: the maximum number of iterations was 250,000 , the initial learning rate of 0.001 with the decaying rate of 0.1 for $80,000 / 150,000$ iterations, weight decay was 0.00004 , momentum

TABLE 7 | Comparison of the results from ImageNet and PlantNet pre-trained backbones in the cascade detector.

\begin{tabular}{lcc}
\hline Class & $\begin{array}{c}\text { Average precision of } \\
\text { ImageNet pre-trained } \\
\text { backbone }\end{array}$ & $\begin{array}{c}\text { Average precision of } \\
\text { PlantNet }\end{array}$ \\
\hline Angular leafspot & $88.41 \%$ & $94.55 \%$ \\
Anthracnose fruit rot & $90.23 \%$ & $96.01 \%$ \\
Gray mold & $90.17 \%$ & $94.52 \%$ \\
Leaf blight & $89.81 \%$ & $91.82 \%$ \\
Leaf spot & $85.16 \%$ & $87.83 \%$ \\
Powdery mildew fruit & $84.01 \%$ & $86.36 \%$ \\
Powdery mildew leaf & $88.59 \%$ & $90.48 \%$ \\
mAP & $88.05 \%$ & $91.65 \%$
\end{tabular}

rate of 0.9. During the training, OHEM (Online Hard Example Mining) was adopted to effectively relieve the class imbalance problem and decrease the false positive error.

\section{Evaluation Method}

The performance of the proposed model was evaluated based on average precision (AP) introduced by the Pascal VOC Challenge (Everingham et al., 2010). AP is the area under the precision and recall curve for the detection task, and has a constant recall level of 0 to 1 . The equation for AP is as follows:

$$
\begin{gathered}
A \boldsymbol{P}=\frac{\mathbf{1}}{\mathbf{1 1}} \sum_{r \in\{\mathbf{0 , 0 . 1 , 0 . 2 , \ldots , 1 \}}} \boldsymbol{P}_{\text {interp }}(r) \\
P_{\text {interp }}(r)=\max _{\tilde{r}: \tilde{r} \geq r} P(\tilde{r})
\end{gathered}
$$

where $P_{\text {interp }}(r)$ and $P(\tilde{r})$ represent the maximum precision for any recall values greater than $r$, and the measured precision of recall, $\tilde{r}$, respectively. The intersection over union (IoU) defined in Equation 3, is a used method for evaluating the detector accuracy:

$$
\operatorname{IoU}(A, B)=\left|\frac{A \cap B}{A \cup B}\right|
$$

where $A$ Brepresent the ground truth box collected in the annotation and $B$ represents the predicted result of the network. If the estimated IoU was higher than a given threshold, the predicted results were considered as positive samples (TP + FP) otherwise they were considered negatives $(\mathrm{FN}+\mathrm{TN})$. IoU is a parameter where the bounding box detected is used to identify True Positive(TP), True Negative(TN), False Positive(FP), and False Negative(FN).

\section{EXPERIMENT RESULTS AND DISCUSSION}

\section{ImageNet and PlantNet Pre-trained Backbone in a Single Stage Detector}

To determine the effect of PlantNet on performance, we compared two different types of backbone feature extractors in a single-stage detector; one was a ResNet152 pre-trained only on the ImageNet dataset and the other was a ResNet152 pretrained on a PlantNet obtained from the LifeCLEF 2017 task. For both experiments, the single-stage detectors were adjusted and fine-tuned with the strawberry training dataset. With the same detection threshold of 0.5 for the FPN, pre-training with ImageNet resulted in $83.13 \% \mathrm{mAP}$, while pre-training from PlantNet resulted in $86.4 \% \mathrm{mAP}$. This result clearly shows how direct use of a PlantNet pre-trained backbone is superior to an ImageNet. PlantNet is capable of capturing domain knowledge prior to being fine-tuned with a small amount of domain-specific data on strawberry diseases. Table 5 summarizes the results of the experiment.

\section{ImageNet and PlantNet Pre-trained Backbones in a Cascade Detector}

In the experiment, we constructed a cascade detector with two different types of backbone feature extractor; one pre-trained 

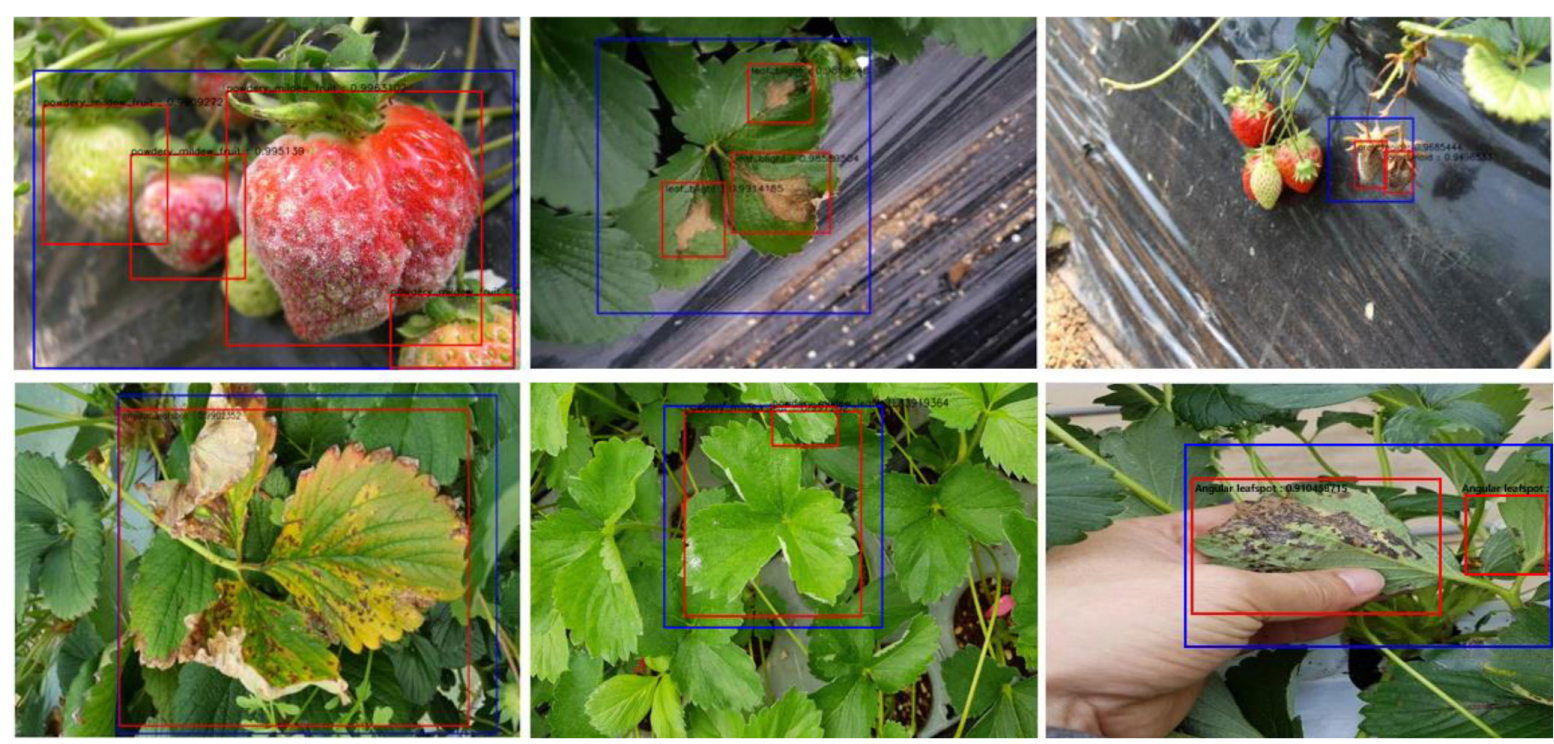

FIGURE 7 | Samples of the final detected results.
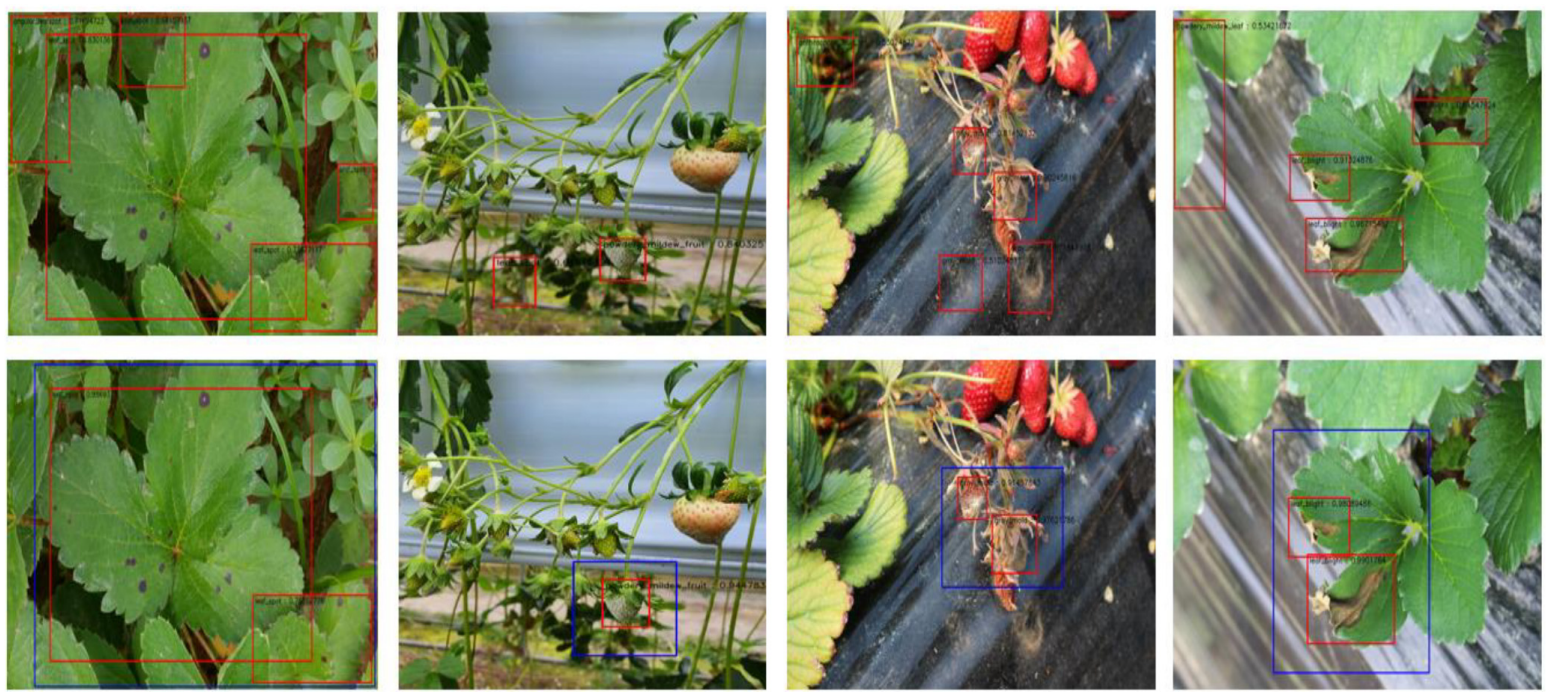

FIGURE 8 | Comparison of the detection results between the single stage (upper row) and two-stage cascade detector (lower row).

with the ImageNet dataset, and the other pre-trained with PlantNet. The number of bounding boxes detected by the object detector decreased as the IoU threshold increased. However, the impact on the cascade structure should also be considered in terms of the recall rate, which is equal to TP/(TP $+\mathrm{FN})$. Table 6 shows true positive, false negative and recall for the IoU detection threshold in the first stage.

IoU detection threshold for the second stage was 0.5 in order to increase precision. The overall mAPs for the ImageNet was $88.05 \%$ and $91.65 \%$ for the PlantNet pre-trained backbones, which are at least $4.92 \%$ and $5.25 \%$ higher than those from the single-stage detector.
The average time until disease detection was $0.241 \mathrm{~s}$ for the single stage cascade detector and $0.662 \mathrm{~s}$ for the twostage cascade detector, using the computation resources described in Section "Training the Strawberry Disease Detector." This shows that the cascade structure improves accuracy, though it comes at the cost of increased detection time. In order to speed up the detection process, pipeline processing could be adopted with our cascade approach. Table 7 summarizes our results, and Figure 7 shows samples of our detection tests, in which blue rectangles represent the merged suspicious areas of disease, and the red boxes are the diseases detected in the second stage. Figure 8 compares the 
results of the single stage and the cascade detectors. The false positive errors in the upper row of the single stage detector are effectively reduced in the lower row of the two-stage cascaded detector.

It is difficult to compare the performance of our proposed method using relative terms, as we have attempted to detect different set of diseases with different data than other researchers. For example, (Nie et al., 2019) have achieved 99.95\% accuracy in identifying four diseases, while others (Kusumandari et al., 2019; Shin et al., 2020) have sought to identify only a single disease.

\section{CONCLUSION}

Protecting plants from diseases is critical to maximizing farm productivity and achieving higher crop quality. The earlier plant diseases can be detected, the more effective and targeted an intervention can be. We reviewed recent research into vision-based plant disease identification, especially research that involved use of DL. We then categorized these interventions into two types based on the degree of human intervention: the 'classification' and 'detection' approaches.

After determining that the detection approach was superior, we proposed an improved method of vision-based detection of strawberry diseases using a DNN that is capable of being incorporated into an automated robot system. In our approach, the backbone feature extractor PlantNet, which was pre-trained on plant data like the PlantCLEF dataset for the LifeCLEF 2017 challenge, was installed with a two-stage cascade disease detection model. PlantNet captured plant domain information quite well, and it demonstrated performance superior to that of the backbone pre-trained on an ImageNet-type public dataset by at least 3.2\% mAP. The cascade detector also improved accuracy by up to $5.25 \% \mathrm{mAP}$. The results indicate that PlantNet is one way to overcome a lack of annotated data by applying plant domain knowledge, and that the human-like cascade detection strategy is effective at improving accuracy.

Diseases and abiotic stresses in the strawberry plant occur everywhere in greenhouses, which is related to environmental data such as low or high temperature, deficient or excessive water, high salinity, heavy metals, and ultraviolet radiation. These

\section{REFERENCES}

Barbedo, J. G. A. (2018). Impact of dataset size and variety on the effectiveness of deep learning and transfer learning for plant disease classification. Comput. Electron. Agric. 153, 46-53.

Brahimi, M., Boukhalfa, K., and Moussaoui, A. (2017). Deep learning for tomato diseases: classification and symptoms visualization. Appl. Artif. Intellig. 31, 299-315.

Cruz, A. C., Luvisi, A., De Bellis, L., and Ampatzidis, Y. (2017). X-FIDO: an effective application for detecting olive quick decline syndrome with deep learning and data fusion. Front. Plant Sci. 8:1741. doi: 10.3389/fpls.2017.01741

Dai, J., Li, Y., He, K., and Sun, J. (2016). "R-fcn: object detection via region-based fully convolutional networks," in Advances in Neural Information Processing Systems, eds T. G. Dietterich, S. Becker, and Z. Ghahramani (London: MIT Press), 379-387. factors are hostile to plant growth and development, leading to crop yield penalty (He et al., 2018). For this reason, pursuing even more accurate, quick, and practical strawberry plant disease and abiotic stress lesion detection techniques is necessary. It can also be helpful to improve the visual analysis results by integrating other environmental data, such as humidity, temperature, and nutrition. In the future development of the proposed method, we will continuously collect environmental and strawberry plant image data for the disease and abiotic stress and can improve the detection accuracy by fusion of visual detection results and environmental knowledge information.

\section{DATA AVAILABILITY STATEMENT}

The original contributions presented in the study are included in the article/supplementary material, further inquiries can be directed to the corresponding author/s.

\section{AUTHOR CONTRIBUTIONS}

BK and JL designed the study, performed the experiments, data analysis, and wrote the manuscript. JL advised on the design of the model and analyzed to find the best method for Improve detection accuracy of diseases of strawberry plants. Y-KH and J-HP collected the data from the greenhouse and contributed the information for the data annotation.

\section{FUNDING}

This work was supported by Korea institute of Planning and Evaluation for Technology in Food, Agriculture and Forestry (IPET) through Smart Plant Farming Industry Technology Development Program, funded by ministry of Agriculture, Food and Rural Affairs(MAFRA) (320089-01).

\section{ACKNOWLEDGMENTS}

This manuscript was proofread by the Writing Center at Jeonbuk National University in November 2020.

Dalal, N., and Triggs, B. (2005). "Histograms of oriented gradients for human detection," in Proceedings of the 2005 IEEE computer society conference on computer vision and pattern recognition (CVPR'05), Vol. 1, (Piscataway, NJ: IEEE), 886-893.

Everingham, M., Van Gool, L., Williams, C. K., Winn, J., and Zisserman, A. (2010). The pascal visual object classes (voc) challenge. Int. J. Comput. Vis. 88, 303-338. doi: 10.1007/s11263-009-0275-4

Ferentinos, K. P. (2018). Deep learning models for plant disease detection and diagnosis. Comput. Electron. Agric. 145, 311-318.

Fuentes, A., Yoon, S., Kim, S. C., and Park, D. S. (2017). A robust deep-learningbased detector for real-time tomato plant diseases and pests recognition. Sensors 17:2022. doi: 10.3390/s17092022

Fuentes, A., Yoon, S., and Park, D. S. (2019). Deep learning-based phenotyping system with glocal description of plant anomalies and symptoms. Front. Plant Sci. 10:1321. doi: 10.3389/fpls.2019.01321 
Fuentes, A. F., Yoon, S., Lee, J., and Park, D. S. (2018). High-performance deep neural network-based tomato plant diseases and pests diagnosis system with refinement filter bank. Front. Plant Sci. 9:1162. doi: 10.3389/fpls.2018. 01162

Girshick, R. (2015). "Fast r-cnn," in Proceedings of the IEEE International Conference on Computer Vision, (Cham: Springer), 1440-1448.

Girshick, R., Donahue, J., Darrell, T., and Malik, J. (2014). "Rich feature hierarchies for accurate object detection and semantic segmentation," in Proceedings of the IEEE Conference on Computer Vision and Pattern Recognition, (Cham: Springer), 580-587.

He, M., He, C.-Q., and Ding, N.-Z. (2018). Abiotic stresses: general defenses of land plants and chances for engineering multistress tolerance. Front. Plant Sci. 9:1771. doi: 10.3389/fpls.2018.01771

Hughes, D. P., and Salathé, M. (2015). An open access repository of images on plant health to enable the development of mobile disease diagnostics. arXiv [Preprint]. Available online at: https://arxiv.org/ftp/arxiv/papers/1511/ 1511.08060.pdf (accessed March 27, 2020).

Joly, A., Goëau, H., Glotin, H., Spampinato, C., Bonnet, P., Vellinga, W. P., et al. (2017). "Lifeclef 2017 lab overview: multimedia species identification challenges," in Proceedings of the International Conference of the Cross-Language Evaluation Forum for European Languages, (Cham: Springer), 255-274. doi: 10.1007/978-3-319-65813-1_24

Kawasaki, Y., Uga, H., Kagiwada, S., and Iyatomi, H. (2015). "Basic study of automated diagnosis of viral plant diseases using convolutional neural networks," in Proceedings of the International Symposium on Visual Computing, (Cham: Springer), 638-645.

Krizhevsky, A., Sutskever, I., and Hinton, G. E. (2012). Imagenet classification with deep convolutional neural networks. Adv. Neural Inform. Proc. Syst. 60:e3065386.

Kusumandari, D. E., Adzkia, M., Gultom, S. P., Turnip, M., and Turnip, A. (2019). Detection of strawberry plant disease based on leaf spot using color segmentation. J. Phys. Conf. Ser. 1230:012092. IOP Publishing, doi: 10.1088/ 1742-6596/1230/1/012092

Lin, T. Y., Dollár, P., Girshick, R., He, K., Hariharan, B., and Belongie, S. (2017). "Feature pyramid networks for object detection," in Proceedings of the IEEE Conference on Computer Vision and Pattern Recognition, (Cham: Springer), 2117-2125.

Lin, T. Y., Maire, M., Belongie, S., Hays, J., Perona, P., Ramanan, D., et al. (2014). "Microsoft coco: common objects in context," in Proceedings of the European Conference on Computer Vision, (Cham: Springer), 740-755. doi: 10.1007/9783-319-10602-1_48

Liu, B., Zhang, Y., He, D., and Li, Y. (2018). Identification of apple leaf diseases based on deep convolutional neural networks. Symmetry 10:11. doi: 10.3390/ sym 10010011

Liu, W., Anguelov, D., Erhan, D., Szegedy, C., Reed, S., Fu, C. Y., et al. (2016). "Ssd: single shot multibox detector," in Proceedings of the European Conference on Computer Vision, (Cham: Springer), 21-37.

Lowe, D. G. (2004). Distinctive image features from scale-invariant keypoints. Int. J. Comput. Vis. 60, 91-110.

Mohanty, S. P., Hughes, D. P., and Salathé, M. (2016). Using deep learning for image-based plant disease detection. Front. Plant Sci. 7:1419. doi: 10.3389/fpls. 2016.01419

Nie, X., Wang, L., Ding, H., and Xu, M. (2019). Strawberry verticillium wilt detection network based on multi-task learning and attention. IEEE Access 7, 170003-170011. doi: 10.1109/access.2019.2954845
Park, K., ki Hong, Y., hwan Kim, G., and Lee, J. (2018). Classification of apple leaf conditions in hyper-spectral images for diagnosis of Marssonina blotch using mRMR and deep neural network. Comput. Electron. Agric. 148, 179-187. doi: 10.1016/j.compag.2018.02.025

Ramcharan, A., Baranowski, K., McCloskey, P., Ahmed, B., Legg, J., and Hughes, D. P. (2017). Deep learning for image-based cassava disease detection. Front. Plant Sci. 8:1852. doi: 10.3389/fpls.2017.01852

Ramcharan, A., McCloskey, P., Baranowski, K., Mbilinyi, N., Mrisho, L., Ndalahwa, M., et al. (2019). A mobile-based deep learning model for cassava disease diagnosis. Front. Plant Sci. 10:272. doi: 10.3389/fpls.2019.00272

Redmon, J., Divvala, S., Girshick, R., and Farhadi, A. (2016). "You only look once: unified, real-time object detection," in Proceedings of the IEEE Conference On Computer Vision and Pattern Recognition, (Cham: Springer), 779-788.

Ren, S., He, K., Girshick, R., and Sun, J. (2015). "Faster r-cnn: towards realtime object detection with region proposal networks," in Advances in Neural Information Processing Systems, eds T. G. Dietterich, S. Becker, and Z. Ghahramani (London: MIT Press), 91-99.

Sermanet, P., Eigen, D., Zhang, X., Mathieu, M., Fergus, R., and LeCun, Y. (2013). Overfeat: integrated recognition, localization and detection using convolutional networks. ArXiv [Preprint]. Available online at: https://arxiv.org/abs/1312.6229 (accessed March 27, 2020).

Shin, J., Chang, Y. K., Heung, B., Nguyen-Quang, T., Price, G. W., and AiMallahi, A. (2020). Effect of directional augmentation using supervised machine learning technologies: a case study of strawberry powdery mildew detection. Biosyst. Eng. 194, 49-60.

Sladojevic, S., Arsenovic, M., Anderla, A., Culibrk, D., and Stefanovic, D. (2016). Deep neural networks based recognition of plant diseases by leaf image classification. Comput. Intellig. Neurosci. 2016:3289801.

Too, E. C., Yujian, L., Njuki, S., and Yingchun, L. (2019). A comparative study of fine-tuning deep learning models for plant disease identification. Comput. Electron. Agric. 161, 272-279. doi: 10.1016/j.compag.2018.03.032

Uijlings, J. R., Van De Sande, K. E., Gevers, T., and Smeulders, A. W. (2013). Selective search for object recognition. Int. J. Comput. Vis. 104, $154-171$.

Viola, P., and Jones, M. (2001). "Rapid object detection using a boosted cascade of simple features," in Proceedings of the 2001 IEEE Computer Society Conference on Computer Vision and Pattern Recognition. CVPR 2001, (Piscataway, NJ: IEEE).

You, J., and Lee, J. (2020). An offline mobile diagnosis system for citrus pests and diseases using deep compression neural network. IET Comput. Vis. 14, 370-377. doi: 10.1049/iet-cvi.2018.5784

Zhao, Z. Q., Zheng, P., Xu, S. T., and Wu, X. (2019). Object detection with deep learning: a review. IEEE Trans. Neural Netw. Learn. Syst. 30, 3212-3232.

Conflict of Interest: The authors declare that the research was conducted in the absence of any commercial or financial relationships that could be construed as a potential conflict of interest.

Copyright (c) $2021 \mathrm{Kim}$, Han, Park and Lee. This is an open-access article distributed under the terms of the Creative Commons Attribution License (CC BY). The use, distribution or reproduction in other forums is permitted, provided the original author(s) and the copyright owner(s) are credited and that the original publication in this journal is cited, in accordance with accepted academic practice. No use, distribution or reproduction is permitted which does not comply with these terms. 